\title{
Transglutaminase 2 in cartilage homoeostasis: novel links with inflammatory osteoarthritis
}

\author{
M. Adamczyk ${ }^{1,2}$ (i)
}

Received: 16 May 2016 / Accepted: 28 July 2016 / Published online: 10 August 2016

(C) The Author(s) 2016. This article is published with open access at Springerlink.com

\begin{abstract}
Transglutaminase 2 (TG2) is highly expressed during chondrocyte maturation and contributes to the formation of a mineralised scaffold by introducing crosslinks between extracellular matrix (ECM) proteins. In healthy cartilage, TG2 stabilises integrity of ECM and likely influences cartilage stiffness and mechanistic properties. At the same time, the abnormal accumulation of TG2 in the ECM promotes chondrocyte hypertrophy and cartilage calcification, which might be an important aspect of osteoarthritis (OA) initiation. Although excessive joint loading and injuries are one of the main causes leading to OA development, it is now being recognised that the presence of inflammatory mediators accelerates OA progression. Inflammatory signalling is known to stimulate the extracellular TG2 activity in cartilage and promote TG2-catalysed crosslinking of molecules that promote chondrocyte osteoarthritic differentiation. It is, however, unclear whether TG2 activity aims to resolve or aggravate damages within the arthritic joint. Better understanding of the complex signalling pathways linking inflammation with TG2 activities is needed to identify the role of
\end{abstract}

Handling Editors: S. Beninati, M. Piacentini, C.M. Bergamini.

M. Adamczyk

adamczykm@cardiff.ac.uk; m.adamczyk@sheffield.ac.uk

1 Matrix Biology and Tissue Repair Research Unit, Oral and Biomedical Sciences, College of Biomedical and Life Sciences, School of Dentistry, Cardiff University, Heath Park, Cardiff CF14 4XY, UK

2 Academic Unit of Bone Biology, Department of Oncology and Metabolism, Mellanby Centre For Bone Research, Medical School, The University of Sheffield, Beech Hill Road, Sheffield S10 2RX, UK
TG2 in OA and to define possible avenues for therapeutic interventions.

Keywords Transglutaminase · Osteoarthritis · Cartilage · Inflammation · Chondrocyte hypertrophy

\section{Introduction}

Osteoarthritis (OA) remains the major disease causing joint degeneration and disability worldwide (Cross et al. 2014). OA is a strongly age-related disorder; however, it can affect young to middle-aged individuals or athletes and have a significant impact on people's lives. Common symptoms of OA include: focal dysregulation within the joint, stiffness, synovial membrane swelling and pain (Goldring and Goldring 2007). The pathogenesis of OA is partly caused by the imbalance between cartilage extracellular matrix (ECM) synthesis and degradation (Goldring and Goldring 2010). The excessive mechanical joint stimulation is one of the main risk factors promoting cartilage surface thinning, erosion and loss of its elastic properties (Ko et al. 2013). The abnormal mechanical stress can lead to chondrocyte differentiation and production of cytokines, aggrecanases and matrix metalloproteinases (MMPs) which disrupt cartilage ECM (Loeser et al. 2012). Cartilage surface wear and chondrocyte apoptosis are often accompanied by subchondral bone adaptive modelling ( $\mathrm{Li}$ et al. 2013) and synovitis (Rahmati et al. 2016). Presence of joint space narrowing, osteophytes, subchondral bone abnormalities or accumulation of the synovial fluid are the clinical features of OA (Bay-Jensen et al. 2010). The diagnosis of $\mathrm{OA}$ is usually confirmed by $\mathrm{X}$-ray, magnetic resonance imaging or arthroscopy. Yet, these structural changes mostly detect late stages of the disease, and too 
little is known about the early mechanism underlying joint degeneration in OA.

Transglutaminase 2 (TG2) is an interesting target involved in OA pathogenesis as accumulation of TG2 in the ECM promotes chondrocyte hypertrophy and cartilage calcification (Tarantino et al. 2011), which mechanism might be linked to early events of the disease. TG2 belongs to the family of transglutaminases (TG), the enzymes that catalyse $\mathrm{Ca}^{2+}$-dependent acyl-transferase reactions (Eckert et al. 2014). Depending on the nature of the substrates available, TG mediate protein crosslinking or small amine incorporation through reaction of transamidation; peptide esterification; or catalyse hydrolysis by deamidation or isopeptide cleavage. The most characteristic reaction mediated by TG2 is that of crosslinking, which results in the formation of covalent $\mathrm{N}^{\varepsilon}(\gamma$-glutamyl)lysyl isopeptide bonds between proteins or within polypeptides (Lorand and Graham 2003). In humans, there are nine TG family members described (Eckert et al. 2014), but the main TG activity in skeletal tissues comes from the activity of TG2 and FXIIIA (Thomázy and Davies 1999; Rosenthal et al. 2001; Deasey et al. 2013). TG1 and TG3 are also detected in the skeletal tissues (Deasey et al. 2013); however, the specific biological functions of TG1 and TG3 in musculoskeletal system remain largely unknown, and this awaits further investigation. In many instances, TG2 is expressed at much higher level than any of the other enzymes of the family, thus can display various functions in disease processes (Iismaa et al. 2009). The predominant role of TG2 is in cell stress response and tissue repair, regulating hallmark events such as ECM remodelling and cell activation (Stephens et al. 2004). Yet, aberrant TG2 activity may also promote formation of protein modifications that can lead to tissue fibrosis (Verderio et al. 2004; Collighan and Griffin 2009). TG2 also drives the destructive immune responses associated with coeliac disease (Sollid and Jabri 2011) and with rheumatoid arthritis (Dzhambazov et al. 2009; Lauzier et al. 2012). Upregulation of TG2 in both OA patient samples as well as in OA animal models has been well established but the main function of TG2 in OA pathogenesis remains enigmatic. Sufficient knowledge of TG2 functions at the initial stages of OA development is needed to understand whether TG2 role is to rescue cartilage damage or promote the irreversible changes within the joint.

Inflammatory signalling stimulates TG2 extracellular crosslinking activities in articular cartilage (Johnson et al. 2001). This is important as the detection of inflammatory molecules in the OA joint in recent years has shed new light for more research into that area (de Lange-Brokaar et al. 2012; Rahmati et al. 2016). Various proinflammatory mediators were found to be elevated in the osteoarthritic joints leading to cartilage destruction, such as interleukins
(IL-1 $\beta$ and IL-6), tumour necrosis factor $\alpha$ (TNF $\alpha$ ), matrix metalloproteinases (MMP 1,3,9 and 13) and aggrecanases (ADAMTS-4 and ADAMTS-5) (Loeser et al. 2012). Tissue markers released by infiltrating macrophages or synoviocytes such as advanced glycation endproducts, high-mobility group box chromosomal protein 1 (Kyostio-Moore et al. 2011), components of the complement system (C3a and C5b-9) (Wang et al. 2012a) are indicative of "microinflammation" taking place in OA joint (Goldring and Berenbaum 2015). Thus, recent evidence suggests that OA should not be considered solely as a degenerative condition as both local inflammation and low-level systemic inflammation play a role in disease progression (Berenbaum 2012; de Lange-Brokaar et al. 2012). The presence of a novel link between TG2 and innate immunity suggests that fast accumulation of extracellular TG2 (Adamczyk et al. 2015) might occur e.g., during joint injury. This review will summarise the most recent findings about the functions of TG2 in healthy and arthritic cartilage, to highlight an important role of TG2 as a potential therapeutic target.

\section{TG2 expression and activity during chondrocyte maturation}

TG2 is expressed and becomes activated at the early stages of long bone development (Aeschlimann and Thomazy 2000). The vast accumulation of TG2 in the cytosol of pre-hypertrophic chondrocytes is followed by TG2 secretion and catalytic activity, which is later detected in the ECM (Aeschlimann et al. 1995). The expression of TG2 diminishes when chondrocytes reach the hypertrophic stage (Aeschlimann et al. 1993). Interestingly, externalisation of TG2 from maturing chondrocytes precedes mineralisation of newly formed ECM (Aeschlimann et al. 1995). It is likely that the retinoids have a significant role in regulating TG2 expression during ossification through binding to RAR and RXR enhancer elements upstream of the TGM2 gene (Nagy et al. 1997). TG-catalysed crosslinks stabilise structure of bone and hyaline cartilage matrix, therefore the presence of enzymatically active TG2 is considered to be an important step in long bone development (Aeschlimann et al. 1995). TG2 might also act as an autocrine factor and control glycosaminoglycans (GAGs) synthesis by regulating the xylosyltransferase 2 transcription during chondrocyte development (Nurminsky et al. 2011). In the pellet cultures of chicken mesenchymal stem cells, the upregulation of TG2 accelerates the transition of chondrocytes into the pre-hypertrophic stage and decreases deposition of extracellular matrix rich in GAGs (Nurminsky et al. 2011). Thus, TG2 plays a role in controlling matrix deposition and crosslinking chondrocyte ECM. 
TG2 protein upregulation does not always correlate with an increase in TG2 catalytic activity. For example, TG2 activity is initially detected in the cartilage anlagen of the spine at E12.5 stage of mouse embryonic development, followed by the increase in the perichondrium (Itoh et al. 2013), which then slowly diminishes despite the TG2 protein expression remaining high. It is possible that the hypoxic environment of cartilage modulates TG2 levels throughout development, as hypoxia is a strong inducer of TG2 expression e.g., in cancer cells (Kumar and Mehta 2012). Clearly, more investigation is needed to underline the mechanisms controlling TG2 expression and activity during development of hyaline, elastic and fibrocartilage.

\section{Regulation of TG2 activity and release in chondrocytes}

TG2 enzymatic activity is allosterically regulated by guanine nucleotides (GTP/GDP) and $\mathrm{Ca}^{2+}$ ions (Achyuthan and Greenberg 1987; Begg et al. 2006). Current understanding suggests that TG2 is rapidly converted from "closed" into the "open" conformation by high extracellular $\mathrm{Ca}^{2+}$ concentrations (Pinkas et al. 2007). On the other hand, GTP/ GDP association promotes transition into more compact conformation (Pinkas et al. 2007). GTP is highly important as molecular manipulation of GTP-binding residues inhibits TG2 externalisation from human chondrocytes (Johnson and Terkeltaub 2005). TG2 can act as an ATPase, however, the affinity for ATP is several orders of magnitude lower compared to GTP (Achyuthan and Greenberg 1987; Schaertl et al. 2010). Hydrolysis of extracellular ATP by TG2 was postulated to be a major function of cell-surface TG2 in osteoblasts, and the hydrolysis product inorganic pyrophosphate is likely involved in promoting mineralisation (Nakano et al. 2007). Recently, it was shown that chondrocytes secrete articular cartilage matrix vesicles containing mRNA for TG2 and FXIIIA, together with ANK, type II collagen, GAPDH and aggrecan (Mitton et al. 2009). Better understanding of TG2 externalisation from chondrocytes would tell us more about its localisation and enzymatic activity in the specific domains of cartilage ECM.

\section{TG2-ECM interactions in articular cartilage}

Chondrocyte cell surface and cartilage ECM is rich in potential binding partners for TG2. These include fibronectin (FN), collagens of type II, III, V and XI, osteopontin, osteonectin, fibrillin, laminin, syndecan-4, integrins, MMP-2 and many others (Lorand and Graham 2003; Belkin 2011). Some of the similar substrates can be targeted by another transglutaminase, FXIIIA (Muszbek et al.
2011). However, it was shown that TG2 and FXIIIA display a preference for distinct sites within the same molecular targets (Watanabe et al. 2013). In many instances, the interactions are independent of TG2 transamidation activity. For instance, TG2 binds FN at the $\mathrm{I}_{6} \mathrm{II}_{1,2} \mathrm{I}_{7-9}$ modules localised in the gelatin-binding region of $\mathrm{FN}$, and this interaction restricts neither TG2 activity nor the interaction of FN with integrins (Gaudry et al. 1999). TG2 was also shown to associate with heparan sulphate chains of syndecan-4, without causing changes in TG2 conformation and/ or affecting TG2/FN interactions (Scarpellini et al. 2009). TG2 interaction with syndecan-4 leads to protein kinase $\mathrm{C}-\alpha$ activation (Telci et al. 2008) and results in inside-out signalling through $\beta 1$ integrin. Although little is known about interaction of TG2 with type II collagen, it is likely that transamidation of TG2 affects the alignment of collagen fibrils and their shape as shown for corneal stroma (Wang et al. 2014). This interaction might be potentially important in mediating mechanistic properties and shear stiffness of articular cartilage.

It is likely that proteoglycans influence TG2 localisation and activity in cartilage ECM. In other connective tissues, one of the best studied examples is TG2 interaction with heparan sulphate chains on syndecan-4. Wang et al. (2012b) proposed a model, in which TG2-bound to syndecan-4 in its "closed" form is shed from the cell surface by MMPs, which leads to TG2 "opening" and re-gaining of its enzymatic activity (Wang et al. 2012b). It is, however, not known if this mechanism might be accelerated during cartilage degradation in OA. Another group identified clusters of positively charged residues on the heparan sulphate that are accessible for TG2 binding in its compact or "closed" conformation (Lortat-Jacob et al. 2012). The release of TG2 from this complex is achieved by an increase in $\mathrm{Ca}^{2+}$ ions, which reduces TG2 affinity for heparan sulphate (LortatJacob et al. 2012), suggesting that this binding might be compromised during tissue calcification. The in vivo data showing attenuation of TG2 extracellular activity in syndecan- $4^{-l-}$ mice indicate that excessive TG2 externalisation could be abolished by specifically cleaving heparan sulphate chains of syndecan-4 (Scarpellini et al. 2014). Thus, reduction in syndecan-4 expression might have an affect on TG2 levels in cartilage ECM due to lack of TG2 "trapping" by the heparan sulphate chains. Recently, syndecan-4 was shown to be regulating activity of ADAMTS-5 (Echtermeyer et al. 2009), which is one of the primary aggrecanases driving cartilage proteolysis in OA models (Glasson et al. 2005). Therefore, TG2 and syndecan-4 binding might reduce or promote ADAMTS-5 transactivation linked with cartilage destruction in OA. Conclusively, investigating TG2 and proteoglycan relationship in cartilage is an interesting avenue for future studies as these interactions might be important in mediating cartilage ECM fragmentation in inflammatory OA. 
Table 1 Compensation of TG2 expression and activity in $\mathrm{TG}^{-1-}$ mice based on the original data shown by (Deasey et al. 2013). In this study, the detailed analysis of hypertrophic cartilage (knee joint), non-hypertrophic cartilage (sternum) and skeletal muscle (limb,

\begin{tabular}{|c|c|c|c|c|c|}
\hline & \multicolumn{2}{|c|}{ Wild-type mice } & \multicolumn{2}{|l|}{$\mathrm{TG} 2^{-1-}$ mice } & \multirow[t]{2}{*}{ Compensation } \\
\hline & Expression & Activity & Expression & Activity & \\
\hline Hypertrophic cartilage & $\begin{array}{l}\text { TG2 } \\
\text { FXIIIA } \\
\text { TG1 (L) }\end{array}$ & $60 \%$ & $\begin{array}{l}\text { FXIIIA (NC) } \\
\text { TG1 (U) } \\
\text { TG3 (U) }\end{array}$ & $80 \%$ & $\begin{array}{l}\text { Yes (to some extent at expression } \\
\text { and activity levels) }\end{array}$ \\
\hline Non-hypertrophic cartilage & $\begin{array}{l}\text { TG2 } \\
\text { FXIIIA } \\
\text { TG1 (L) } \\
\text { TG3 (L) }\end{array}$ & $100 \%$ & $\begin{array}{l}\text { FXIIIA (NC) } \\
\text { TG1 (D) } \\
\text { TG3 (ND) }\end{array}$ & $3 \times$ increase & Yes (only at activity level) \\
\hline Skeletal muscle & $\begin{array}{l}\text { TG2 } \\
\text { FXIIIA } \\
\text { TG1 (L) }\end{array}$ & $60 \%$ & $\begin{array}{l}\text { FXIIIA (D) } \\
\text { TG1 (NC) }\end{array}$ & $40 \%$ & Lack \\
\hline
\end{tabular}

This table was prepared based on the original data published by Deasey et al. (2013)

$L$ low expressed, $N C$ not changed, $U$ upregulated, $D$ downregulated, $N D$ not detected

\section{Lessons from TG2 $^{-1-}$ animals}

Despite the important role of TG2 in cartilage and bone development, $\mathrm{TG} 2^{-/-}$mice have no developmental abnormalities (De Laurenzi and Melino 2001; Nanda et al. 2001). The lack of obvious defects in TG2-deficient mice may be explained by the activity of other TGs (mainly FXIIIA) that partially compensate the absence of TG2 (see Table 1) (Deasey et al. 2013). Many studies have been conducted with use of $\mathrm{TG} 2^{-1-}$ mice, and, collectively, these show that deficiencies become apparent once the mice are subjected to different types of insults (Oh et al. 2011). The inflammatory response in TG $2^{-l-}$ mice is substantially different. This is manifested by an increase in IL-1 $\beta$ release and higher neutrophil accumulation in response to monosodium urate crystals (MSU) crystal injection (Yen et al. 2015). Also slower clearance of apoptotic neutrophils is linked to reduced ability of macrophage to effectively phagocytose cell debris (Rose et al. 2006). Similarly, there is a significant increase in number of apoptotic cells in thymus of $\mathrm{TG}^{-} /^{-}$mice treated with dexamethasone and accelerated cell death of $\mathrm{TG}^{-} /^{-}$thymocytes exposed to apoptotic signalling (Nanda et al. 2001). This indicates that crosslinking of cellular content by TG2 might become important in controlling cell viability and apoptotic cell clearance especially in early inflammatory processes.

\section{TG2 upregulation in OA cartilage}

In the healthy knee joint, TG2 is mainly expressed in the superficial and deep zone of cartilage, as well as in the chondrocytic part of the menisci (Johnson et al. 2001). In the Hartley guinea pig model that spontaneously develops OA, TG2 expression in cartilage changes during disease aorta, heart, kidney and liver) was performed to compare the TG gene expression and transamidation activity between wild-type and $\mathrm{TG} 2^{-I-}$ mice 

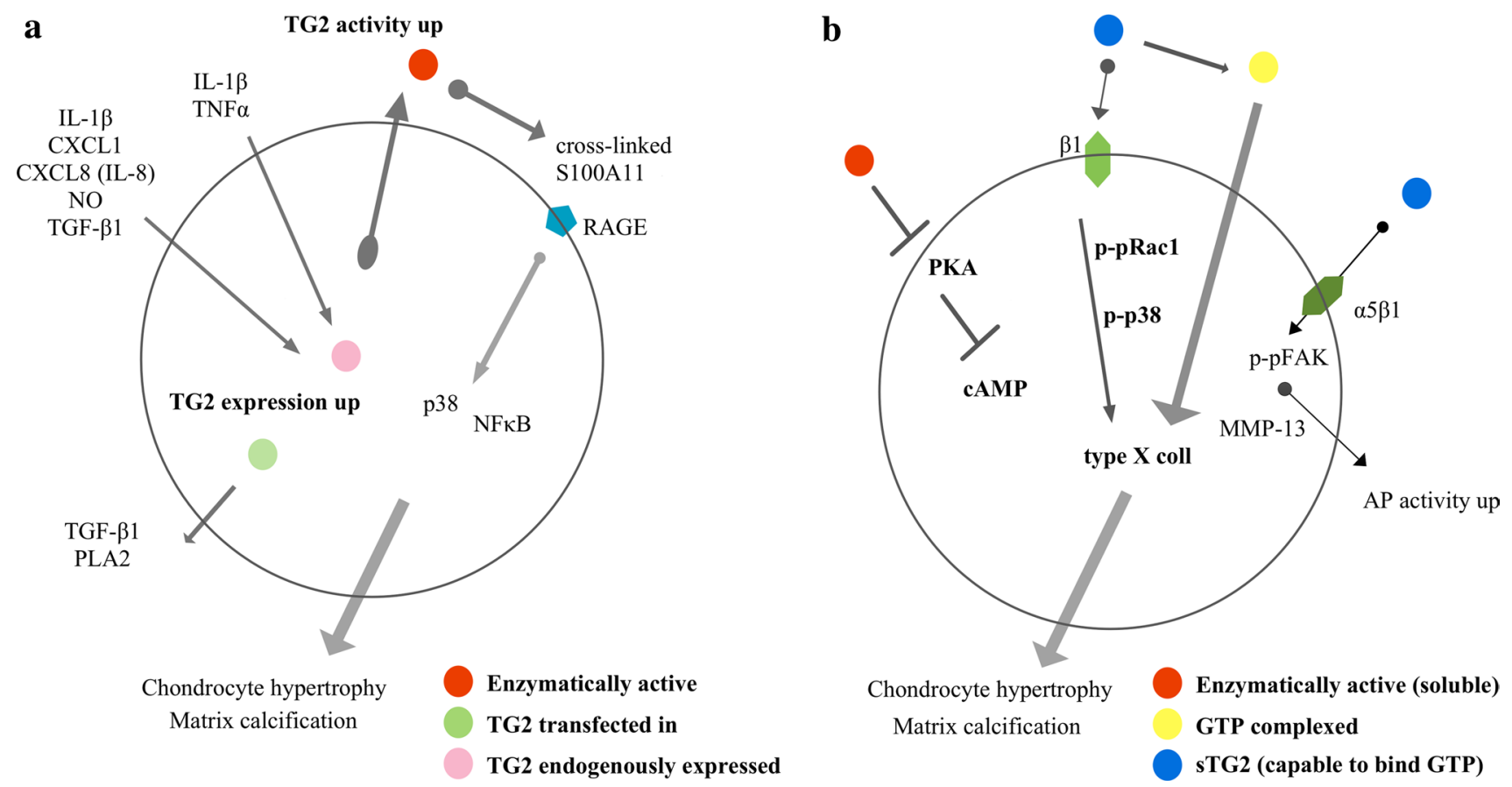

Fig. 1 Summary of TG2-driven "inside-out" (a) and "outside-in" (b) signalling linked with chondrocyte osteoarthritic differentiation

synovial or cell-associated TG2 may be a potentially useful marker of disease progression.

\section{Inside-out and outside-in signalling mediated by TG2 in OA}

TG2 is involved in the pathologic cartilage calcification through inside-out and outside-in signalling (Fig. 1). Mineralisation of cartilage is mediated by hypertrophic chondrocytes, which fuse and enlarge during OA progression (Goldring 2000). Treatment of chondrocytes with all-trans retinoic acid (ATRA) stimulates TG2 expression and potentiates chondrocyte re-differentiation from resting state into hypertrophic or "OA-like" chondrocytes (Johnson et al. 2001). Alongside TG2 induction, ATRA promotes expression of hypertrophic markers such as alkaline phosphatase (AP), collagen type X, or metalloproteinase-13 (MMP-13) suggestive of a direct link (Johnson et al. 2003; Huebner et al. 2009). Interestingly, transient TG2 transfection is sufficient to induce matrix calcification and collagen type $\mathrm{X}$ upregulation in human chondrocytes (Johnson and Terkeltaub 2005) (Fig. 1a). In meniscal chondrocytes, the activity of both TG2 and nucleoside triphosphate pyrophosphohydrolase gradually increases with age (Johnson et al. 2001). During spontaneous OA development in guinea pig, increased TG2 activity positively correlates with the increase of inorganic pyrophosphate and nucleotide pyrophosphatase phosphodiesterase (Johnson et al. 2004).
Several studies demonstrated that FXIIIA transamidation activity stimulates mineralisation of osteoblast cultures (Al-Jallad et al. 2006; Nakano et al. 2010). Likely similar mechanism might be conserved in chondrocytes, whereby increase in TG2 transamidation is involved in pathological cartilage ECM calcification.

The in vitro experiments indicate that soluble TG2 (sTG2) can also act as extracellular alarmin, promoting chondrocyte osteoarthritic differentiation through outsidein signalling (Fig. 1b). Addition of sTG2 to the growth medium of human chondrocytes is sufficient to induce $\beta 1$ integrin signalling that causes phosphorylation of Rac1 and p38 kinase and, ultimately, type $\mathrm{X}$ collagen upregulation (Johnson and Terkeltaub 2005). Moreover, the presence of soluble and enzymatically active TG2 inhibits protein kinase A (PKA) signalling and, ultimately, cyclic adenosine monophosphate (cAMP) production in chondrocytes, also causing matrix calcification (Nurminsky et al. 2011). Interestingly, the stimulation of bovine cartilage explants with sTG2 induces stronger type $\mathrm{X}$ collagen expression when TG2 was in complex with Mg-GTP (Johnson and Terkeltaub 2005). Thus, Johnson and Terkeltaub (2005) proposed an interesting hypothesis suggesting that pathological changes in OA may, at least in part, be due to the presence of an inactive, Mg-GTP-bound form of TG2. In agreement with those findings, stimulation of human chondrocytes with GTP complexed TG2, increases MMP-13 and type $X$ collagen on mRNA levels (Tanaka et al. 2007). The rise in hypertrophic markers associates with elevated 
AP activity and possibly occurs via $\alpha 5 \beta 1$ integrin signalling and Fak phosphorylation. Hence, TG2, particularly in its compact conformation, might be involved in promoting hypertrophic changes and ECM calcification in cartilage.

Once released from the cells, TG2 becomes catalytically active in the extracellular space and catalyses reactions that might promote or contribute to the pathology of OA. TG2mediated crosslinking of the S100A11 molecule allowing formation of $\gamma$-glutamyl- $\varepsilon$-lysine isopeptide bridges turns it into a functional homodimer, which binds more efficiently to the cell surface receptor for advanced glycation end products (RAGE) (Cecil and Terkeltaub 2008). This enhances outside-in signalling, the activation of $\mathrm{p} 38$ MAPK kinase and NFKB signalling pathways, and ultimately induces chondrocyte hypertrophy.

\section{Regulation of TG2 by anabolic and catabolic signalling}

The anabolic signalling mediated by transforming growth factor $\beta 1$ (TGF- $\beta 1)$ is an important mechanism controlling TG2 levels in cartilage and bone. TGF- $\beta 1$ seems to be a slightly better inducer of TG2 protein expression than IL-1 $\beta$ but has no effect on elevating TG2 activity (Johnson et al. 2001). TG2 has been proposed to have a role in TGF- $\beta 1$ activation (Szondy et al. 2003), and an increased expression of TGF- $\beta 1$ in TG $2^{-1-}$ mice was reported (Tarantino et al. 2009). Therefore, TG2 in conjunction with TGF- $\beta 1$ might be involved in ameliorating the inflammatory responses in the resolution phase.

The catabolic responses mediated by chemokine CXCL1 and CXCL8 (IL-8) are implicated in promoting aberrant TG2 activity (Merz et al. 2003). Also, treatment with proinflammatory IL-1 $\beta$ causes secretion of TG2 in mouse articular chondrocytes or bovine cartilage explants (Johnson et al. 2003; Cecil and Terkeltaub 2008). Similarly, patient-derived meniscal cells effectively upregulate TG2 activity in the presence of IL-1 $\beta$ (Johnson et al. 2001). It is likely that nitric oxide (NO) released upon IL-1 $\beta$ priming is responsible for promoting TG2 expression as peroxynitrite donors significantly enhance TG2 activity in a similar manner. TG2 seems to be the predominant TG responding to early catabolic signalling as IL-1 $\beta$ stimulation fails to induce transamidation activity of FXIIIA in cartilage (Johnson et al. 2003).

\section{Role of TG2 in gout and rheumatoid arthritis}

Although the aetiology of OA is different than gout or rheumatoid arthritis, some of the mechanisms regulating TG2 might be common and exist in inflammatory type of OA. Gout is a chronic inflammatory arthritis that is characterised by the deposition of monosodium urate crystals (MSU) usually within the metatarsophalangeal, midfoot and ankle joints (Dalbeth et al. 2016). The TG2 mRNA levels are clearly enhanced in the synovial fluid collected from gouty but not from rheumatoid arthritis patients (Yen et al. 2015). Stimulation of cells with MSU crystals alone causes an enhanced TG2 mRNA expression in primary peripheral blood monocytes or macrophages (Yen et al. 2015). It is likely that TG2 activity reduces the release of proinflammatory IL- $1 \beta$ and TNF $\alpha$ upon MSU exposure by enhancing production of anti-inflammatory TGF- $\beta 1$ (Yen et al. 2015). Yet another study suggested that TG2 transamidation activity is not necessary to increase macrophage phagocytosis by TGF- $\beta 1$ but be rather regulated by TG2 binding to the nucleotides, particularly GDP, ATP or ADP (Rose et al. 2006).

Interesting evidence was reported about the role of TG2 activity in rheumatoid arthritis (RA). RA is a chronic, autoimmune disease, in which synovial inflammation drives destruction of cartilage and bone by activated blood cells infiltrating joint space (Pratt et al. 2009). Induction of experimental arthritis by type II collagen immunization increases TG2 activity in the areas of cartilage erosion and within inflammed synovial membrane (Lauzier et al. 2012). The arthritic fibroblast-like synoviocytes were shown to release enzymatically active TG2 at the sites of collagen type II degradation. Importantly, TG2 was suggested to be actively involved in development of autoimmunity due to its ability to modify an immunodominant fragment of type II collagen (CII260-270) (Dzhambazov et al. 2009). TG2 crosslinking and/or deamidation of this epitope mediates a T-cell response to the CII260-270 fragment (Dzhambazov et al. 2009). Thus, TGs activity in RA animal models can enhance joint destruction by worsening the severity and histopathological features of the disease.

\section{Conclusions}

This review demonstrates that TG2 is an interesting target in inflammatory OA pathogenesis, as there is strong evidence both from human and animal models for the involvement of TG2 in OA progression. The literature suggests that aberrant TG2 upregulation in the arthritic joint might be aggravating OA severity by promoting tissue mineralisation, accelerating chondrocyte hypertrophy and potentially enhancing OA-linked signalling. Likewise, recent findings indicate that FXIIIA might be an important player in inflammatory arthritis, as FXIIIA ${ }^{-/}$mice are protected from cartilage and bone destruction, which might be due to reduced osteoclasts fusion and activity (Raghu et al. 2015). Interestingly, treatment of arthritic joints with inhibitor cystamine to block FXIIIA activity significantly ameliorated type II collagen-induced 
arthritis severity in mice (Raghu et al. 2015). TG2 could also be involved as cystamine displays high specificity towards inhibiting TG2 transamination activity (Siegel and Khosla 2007). Future studies should, therefore, address TG2 functions within inflamed OA joint, taking into account the role of synovial membrane and subchondral bone into consideration despite evidence suggesting TG2 mainly as an appropriate marker of hypertrophic chondrocytes but not upregulated in the remodelled trabecular bone of OA patients (Tarquini et al. 2016). Recent findings demonstrating the role of $P 2 X 7$ receptor-dependent pore formation in TG2 externalisation (Adamczyk et al. 2015) might be relevant in the context of TG2 secretion from activated synovial macrophages. This would suggest the presence of a link between innate immunity and TG2 externalisation, especially vital as P2X7 receptor activation drives the inflammatory responses in joint diseases (Labasi et al. 2002; Lopez-Castejon et al. 2010).

Managing and treating musculoskeletal diseases is a big challenge for ageing population, and there are currently no reliable biochemical markers for early patient diagnosis (Mobasheri et al. 2015). Evidence suggests that diagnostic assays based on the detection of proteins or peptides with post-translational modifications may have a higher specificity than assays detecting only changes in protein levels (Doyle and Mamula 2005). TG2 released by chondrocytes in OA could contribute to the elevated levels of $\gamma$-glutamyl$\varepsilon$-lysine crosslinks present in OA tissue. In summary, more research is needed to clarify if specific post-translational modifications are generated by TG2 in certain OA phenotypes that could serve as biological indicators of the disease.

Acknowledgments The author would like to acknowledge Arthritis Research UK for providing Foundation Fellowship funding (20512) and thank Dr Alison Gartland for support and useful comments.

\section{Compliance with ethical standards}

Conflict of interest The author declares no conflict of interest.

Research involving human participants and/or animals This article does not contain any studies with human participants or animals performed by any of the authors.

Open Access This article is distributed under the terms of the Creative Commons Attribution 4.0 International License (http://creativecommons.org/licenses/by/4.0/), which permits unrestricted use, distribution, and reproduction in any medium, provided you give appropriate credit to the original author(s) and the source, provide a link to the Creative Commons license, and indicate if changes were made.

\section{References}

Achyuthan KE, Greenberg CS (1987) Identification of a guanosine triphosphate-binding site on guinea pig liver transglutaminase. Role of GTP and calcium ions in modulating activity. J Biol Chem 262:1901-1906
Adamczyk M, Griffiths R, Dewitt S et al (2015) P2X7 receptor activation regulates rapid unconventional export of transglutaminase-2. J Cell Sci 128:4615-4628. doi:10.1242/jcs.175968

Aeschlimann D, Thomazy V (2000) Protein crosslinking in assembly and remodelling of extracellular matrices: the role of transglutaminases. Connect Tissue Res 41:1-27. doi: $10.3109 / 03008200009005638$

Aeschlimann D, Wetterwald A, Fleisch H, Paulsson M (1993) Expression of tissue transglutaminase in skeletal tissues correlates with events of terminal differentiation of chondrocytes. J Cell Biol 120:1461-1470. doi:10.1083/jcb.120.6.1461

Aeschlimann D, Kaupp O, Paulsson M (1995) Transglutaminase-catalyzed matrix cross-linking in differentiating cartilage: identification of osteonectin as a major glutaminyl substrate. J Cell Biol 129:881-892. doi:10.1083/jcb.129.3.881

Al-Jallad HF, Nakano Y, Chen JLY et al (2006) Transglutaminase activity regulates osteoblast differentiation and matrix mineralization in MC3T3-E1 osteoblast cultures. Matrix Biol 25:135148. doi:10.1016/j.matbio.2005.11.001

Bay-Jensen AC, Hoegh-Madsen S, Dam E et al (2010) Which elements are involved in reversible and irreversible cartilage degradation in osteoarthritis? Rheumatol Int 30:435-442. doi:10.1007/ s00296-009-1183-1

Begg GE, Carrington L, Stokes PH et al (2006) Mechanism of allosteric regulation of transglutaminase 2 by GTP. Proc Natl Acad Sci U S A 103:19683-19688. doi:10.1073/pnas.0609283103

Belkin AM (2011) Extracellular TG2: emerging functions and regulation. FEBS J 278:4704-4716. doi:10.1111/j.1742-4658.2011.08346.x

Berenbaum F (2012) Osteoarthritis as an inflammatory disease (osteoarthritis is not osteoarthrosis!). Osteoarthr Cartil 21:16-21. doi:10.1016/j.joca.2012.11.012

Cecil DL, Terkeltaub R (2008) Transamidation by transglutaminase 2 transforms S100A11 calgranulin into a procatabolic cytokine for chondrocytes. J Immunol 180:8378-8385. doi:10.4049/ jimmunol.180.12.8378

Collighan RJ, Griffin M (2009) Transglutaminase 2 cross-linking of matrix proteins: biological significance and medical applications. Amino Acids 36:659-670. doi:10.1007/s00726-008-0190-y

Cross M, Smith E, Hoy D et al (2014) The global burden of hip and knee osteoarthritis: estimates from the Global Burden of Disease 2010 study. Ann Rheum Dis 73:1323-1330. doi:10.1136/ annrheumdis-2013-204763

Dalbeth N, Merriman TR, Stamp LK (2016) Gout. Lancet 6736:1-14. doi:10.1016/S0140-6736(16)00346-9

de Lange-Brokaar BJE, Ioan-Facsinay A, van Osch GJVM et al (2012) Synovial inflammation, immune cells and their cytokines in osteoarthritis: a review. Osteoarthr Cartil 20:1484-1499. doi:10.1016/j.joca.2012.08.027

De Laurenzi V, Melino G (2001) Gene disruption of tissue transglutaminase. Mol Cell Biol 21:148-155. doi:10.1128/MCB.21.1.148

Deasey S, Shanmugasundaram S, Nurminskaya M (2013) Tissuespecific responses to loss of transglutaminase 2. Amino Acids 44:179-187. doi:10.1007/s00726-011-1183-9

Doyle HA, Mamula MJ (2005) Posttranslational modifications of self-antigens. Ann N Y Acad Sci 1050:1-9. doi:10.1196/ annals. 1313.001

Dzhambazov B, Lindh I, Engström A, Holmdahl R (2009) Tissue transglutaminase enhances collagen type II-induced arthritis and modifies the immunodominant T-cell epitope CII260-270. Eur J Immunol 39:2412-2423. doi:10.1002/eji.200939438

Echtermeyer F, Bertrand J, Dreier R et al (2009) Syndecan-4 regulates ADAMTS-5 activation and cartilage breakdown in osteoarthritis. Nat Med 15:1072-1076. doi:10.1038/nm.1998

Eckert RL, Kaartinen MT, Nurminskaya M et al (2014) Transglutaminase regulation of cell function. Physiol Rev 94:383-417. doi:10.1152/physrev.00019.2013 
Gaudry CA, Verderio E, Jones RA et al (1999) Tissue transglutaminase is an important player at the surface of human endothelial cells: evidence for its externalization and its colocalization with the beta(1) integrin. Exp Cell Res 252:104-113. doi:10.1006/ excr.1999.4633

Glasson SS, Askew R, Sheppard B et al (2005) Deletion of active ADAMTS5 prevents cartilage degradation in a murine model of osteoarthritis. Nature 434:644-648. doi:10.1038/nature03453.1

Goldring MB (2000) The role of the chondrocyte in osteoarthritis. Arthritis Rheum 43:1916-1926. doi:10.1002/1529-0131(200009)

Goldring MB, Berenbaum F (2015) Emerging targets in osteoarthritis therapy. Curr Opin Pharmacol 22:51-63. doi:10.1016/j. coph.2015.03.004

Goldring MB, Goldring SR (2007) Osteoarthritis. J Cell Physiol 213:626-634. doi:10.1002/jcp.21258

Goldring MB, Goldring SR (2010) Articular cartilage and subchondral bone in the pathogenesis of osteoarthritis. Ann N Y Acad Sci 1192:230-237. doi:10.1111/j.1749-6632.2009.05240.x

Huebner JL, Johnson KA, Kraus VB, Terkeltaub RA (2009) Transglutaminase 2 is a marker of chondrocyte hypertrophy and osteoarthritis severity in the Hartley guinea pig model of knee OA. Osteoarthr Cartil 17:1056-1064. doi:10.1016/j.joca.2009.02.015

Iismaa SE, Mearns BM, Lorand L, Graham RM (2009) Transglutaminases and disease: lessons from genetically engineered mouse models and inherited disorders. Physiol Rev 89:991-1023. doi:10.1152/physrev.00044.2008

Itoh M, Tatsukawa H, Eun-Seo L et al (2013) Variations in both TG1 and TG2 isozyme-specific in situ activities and protein expressions during mouse embryonic development. J Histochem Cytochem 61:793-801. doi:10.1369/0022155413501676

Johnson KA, Terkeltaub RA (2005) External GTP-bound transglutaminase 2 is a molecular switch for chondrocyte hypertrophic differentiation and calcification. J Biol Chem 280:15004-15012. doi:10.1074/jbc.M500962200

Johnson K, Hashimoto S, Lotz M et al (2001) Interleukin-1 induces pro-mineralizing activity of cartilage tissue transglutaminase and factor XIIIa. Am J Pathol 159:149-163. doi:10.1016/ S0002-9440(10)61682-3

Johnson KA, van Etten D, Nanda N et al (2003) Distinct transglutaminase 2-independent and transglutaminase 2-dependent pathways mediate articular chondrocyte hypertrophy. J Biol Chem 278:18824-18832. doi:10.1074/jbc.M301055200

Johnson K, Svensson CI, Van Etten D et al (2004) Mediation of spontaneous knee osteoarthritis by progressive chondrocyte ATP depletion in Hartley guinea pigs. Arthritis Rheum 50:1216-1225. doi:10.1002/art.20149

Ko FC, Dragomir C, Plumb DA et al (2013) In Vivo cyclic compression causes cartilage degeneration and subchondral bone changes in mouse tibiae. Arthritis Rheum 65:1569-1578. doi:10.1002/ art.37906

Kumar S, Mehta K (2012) Tissue transglutaminase constitutively activates HIF- $1 \alpha$ promoter and nuclear factor- $\kappa \mathrm{B}$ via a noncanonical pathway. PLoS One 7:e49321. doi:10.1371/journal. pone.0049321

Kyostio-Moore S, Nambiar B, Hutto E et al (2011) STR/ort mice, a model for spontaneous osteoarthritis, exhibit elevated levels of both local and systemic inflammatory markers. Comp Med 61:346-355

Labasi JM, Petrushova N, Donovan C et al (2002) Absence of the $\mathrm{P} 2 \mathrm{X} 7$ receptor alters leukocyte function and attenuates an inflammatory response. J Immunol 168:6436-6445. doi:10.4049/ jimmunol.168.12.6436

Lauzier A, Charbonneau M, Paquette M et al (2012) Transglutaminase 2 cross-linking activity is linked to invadopodia formation and cartilage breakdown in arthritis. Arthritis Res Ther 14:R159. doi:10.1186/ar3899
Li G, Yin J, Gao J et al (2013) Subchondral bone in osteoarthritis: insight into risk factors and microstructural changes. Arthritis Res Ther 15:223. doi:10.1186/ar4405

Loeser RF, Goldring SR, Scanzello CR, Goldring MB (2012) Osteoarthritis: a disease of the joint as an organ. Arthritis Rheum 64:1697-1707. doi:10.1002/art.34453

Lopez-Castejon G, Theaker J, Pelegrin P et al (2010) P2X(7) receptormediated release of cathepsins from macrophages is a cytokineindependent mechanism potentially involved in joint diseases. $\mathrm{J}$ Immunol 185:2611-2619. doi:10.4049/jimmunol.1000436

Lorand L, Graham RM (2003) Transglutaminases: crosslinking enzymes with pleiotropic functions. Nat Rev Mol Cell Biol 4:140-156. doi:10.1038/nrm1014

Lortat-Jacob H, Burhan I, Scarpellini A et al (2012) Transglutaminase- 2 interaction with heparin: identification of a heparin binding site that regulates cell adhesion to fibronectin-transglutaminase-2 matrix. J Biol Chem 287:18005-18017. doi:10.1074/jbc. M111.337089

Merz D, Liu R, Johnson K, Terkeltaub R (2003) IL-8/CXCL8 and growth-related oncogene alpha/CXCL1 induce chondrocyte hypertrophic differentiation. J Immunol 171:4406-4415. doi:10.4049/jimmunol.171.8.4406

Mitton E, Gohr C, McNally M, Rosenthal A (2009) Articular cartilage vesicles contain RNA. Biochem Biophys Res Commun 388:533-538. doi:10.1037/a0030561

Mobasheri A, Henrotin Y (2015) Biomarkers of (osteo)arthritis. Biomarkers 20:513-518. doi:10.3109/1354750X.2016.1140930

Muszbek L, Bereczky Z, Bagoly Z et al (2011) Factor XIII: a coagulation factor with multiple plasmatic and cellular functions. Physiol Rev 91:931-972. doi:10.1152/physrev.00016.2010

Nagy L, Thomázy VA, Saydak MM et al (1997) The promoter of the mouse tissue transglutaminase gene directs tissue-specific, retinoid-regulated and apoptosis-linked expression. Cell Death Differ 4:534-547. doi:10.1038/sj.cdd.4400290

Nakano Y, Addison WN, Kaartinen MT (2007) ATP-mediated mineralization of MC3T3-E1 osteoblast cultures. Bone 41:549-561. doi:10.1016/j.bone.2007.06.011

Nakano Y, Forsprecher J, Kaartinen MT (2010) Regulation of ATPase activity of transglutaminase 2 by MT1-MMP: implications for mineralization of MC3T3-E1 osteoblast cultures. J Cell Physiol 223:260-269. doi:10.1002/jcp.22034

Nanda N, Iismaa SE, Owens WA et al (2001) Targeted inactivation of Gh/tissue transglutaminase II. J Biol Chem 276:20673-20678. doi:10.1074/jbc.M010846200

Nurminsky D, Shanmugasundaram S, Deaseym S et al (2011) Transglutaminase 2 regulates early chondrogenesis and glycosaminoglycan synthesis. Mech Dev 128:234-245. doi:10.1016/j.mod.2010.11.007

Oh K, Park H, Byoun O et al (2011) Epithelial transglutaminase 2 is needed for $\mathrm{T}$ cell interleukin-17 production and subsequent pulmonary inflammation and fibrosis in bleomycin-treated mice. $\mathrm{J}$ Exp Med 208:1707-1719. doi:10.4049/jimmunol.171.10.5470

Orlandi A, Oliva F, Taurisano G et al (2009) Transglutaminase-2 differently regulates cartilage destruction and osteophyte formation in a surgical model of osteoarthritis. Amino Acids 36:755-763. doi:10.1007/s00726-008-0129-3

Pinkas DM, Strop P, Brunger AT, Khosla C (2007) Transglutaminase 2 undergoes a large conformational change upon activation. PLoS Biol 5:e327. doi:10.1371/journal.pbio.0050327

Pratt AG, Isaacs JD, Mattey DL (2009) Current concepts in the pathogenesis of early rheumatoid arthritis. Best Pract Res Clin Rheumatol 23:37-48. doi:10.1016/j.berh.2008.08.002

Raghu H, Cruz C, Rewerts CL et al (2015) Transglutaminase factor XIII promotes arthritis through mechanisms linked to inflammation and bone erosion. Blood 125:427-438. doi:10.1182/ blood-2014-08-594754 
Rahmati M, Mobasheri A, Mozafari M (2016) Inflammatory mediators in osteoarthritis: a critical review of the state-of-the-art, current prospects, and future challenges. Bone 85:81-90. doi:10.1016/j.bone.2016.01.019

Rose DM, Sydlaske AD, Agha-Babakhani A et al (2006) Transglutaminase 2 limits murine peritoneal acute gout-like inflammation by regulating macrophage clearance of apoptotic neutrophils. Arthritis Rheum 54:3363-3371. doi:10.1002/art.22137

Rosenthal AK, Masuda I, Gohr CM et al (2001) The transglutaminase, Factor XIIIA, is present in articular chondrocytes. Osteoarthr Cartil 9:578-581. doi:10.1053/joca.2000.0423

Scarpellini A, Germack R, Lortat-Jacob H et al (2009) Heparan sulfate proteoglycans are receptors for the cell-surface trafficking and biological activity of transglutaminase-2. J Biol Chem 284:18411-18423. doi:10.1074/jbc.M109.012948

Scarpellini A, Huang L, Burhan I et al (2014) Syndecan-4 knockout leads to reduced extracellular transglutaminase-2 and protects against tubulointerstitial fibrosis. J Am Soc Nephrol 25:10131027. doi:10.1681/ASN.2013050563

Schaertl S, Prime M, Wityak J et al (2010) A profiling platform for the characterization of transglutaminase 2 (TG2) inhibitors. J Biomol Screen 15:478-487. doi:10.1177/1087057110366035

Siegel M, Khosla C (2007) Transglutaminase 2 inhibitors and their therapeutic role in disease states. Pharmacol Ther 115:232-245. doi:10.1016/j.pharmthera.2007.05.003

Sollid LM, Jabri B (2011) Celiac disease and transglutaminase 2: a model for posttranslational modification of antigens and HLA association in the pathogenesis of autoimmune disorders. Curr Opin Immunol 23:732-738. doi:10.1016/j.coi.2011.08.006

Stephens P, Grenard P, Aeschlimann P et al (2004) Crosslinking and G-protein functions of transglutaminase 2 contribute differentially to fibroblast wound healing responses. J Cell Sci 117:3389-3403. doi:10.1242/jcs.01188

Szondy Z, Sarang Z, Molnár P et al (2003) Phagocytosis-Associated crosstalk between macrophages and apoptotic cells. Proc Natl Acad Sci U S A 100:7812-7817. doi:10.1073/pnas.0832466100

Tanaka K, Yokosaki Y, Higashikawa F et al (2007) The integrin $\alpha 5 \beta 1$ regulates chondrocyte hypertrophic differentiation induced by GTP-bound transglutaminase 2. Matrix Biol 26:409-418. doi:10.1016/j.matbio.2007.04.005

Tarantino U, Oliva F, Taurisano G et al (2009) FXIIIA and TGFbeta over-expression produces normal musculo-skeletal phenotype in TG2-/- mice. Amino Acids 36:679-684. doi:10.1007/ s00726-008-0133-7

Tarantino U, Ferlosio A, Arcuri G et al (2011) Transglutaminase 2 as a biomarker of osteoarthritis: an update. Amino Acids 44:199207. doi:10.1007/s00726-011-1181-y

Tarquini C, Mattera R, Mastrangeli F et al (2016) Comparison of tissue transglutaminase 2 and bone biological markers osteocalcin, osteopontin and sclerostin expression in human osteoporosis and osteoarthritis. Amino Acids. doi:10.1007/s00726-016-2290-4

Telci D, Wang Z, Li X et al (2008) Fibronectin-tissue transglutaminase matrix rescues RGD-impaired cell adhesion through syndecan-4 and beta1 integrin co-signaling. J Biol Chem 283:2093720947. doi:10.1074/jbc.M801763200

Thomázy VA, Davies PJ (1999) Expression of tissue transglutaminase in the developing chicken limb is associated both with apoptosis and endochondral ossification. Cell Death Differ 6:146-154. doi:10.1038/sj.cdd.4400464

Verderio EA, Johnson T, Griffin M (2004) Tissue transglutaminase in normal and abnormal wound healing: review article. Amino Acids 26:387-404. doi:10.1007/s00726-004-0094-4

Wang Q, Rozelle AL, Lepus CM et al (2012a) Identification of a central role for complement in osteoarthritis. Nat Med 17:16741679. doi: $10.1038 / \mathrm{nm} .2543$

Wang Z, Collighan RJ, Pytel K et al (2012b) Characterization of heparin-binding site of tissue transglutaminase: its importance in cell surface targeting, matrix deposition, and cell signaling. J Biol Chem 287:13063-13083. doi:10.1074/jbc.M111.294819

Wang L, Uhlig PC, Eikenberry EF et al (2014) Lateral growth limitation of corneal fibrils and their lamellar stacking depend on covalent collagen cross-linking by transglutaminase- 2 and lysyl oxidases, respectively. J Biol Chem 289:921-929. doi:10.1074/ jbc.M113.496364

Watanabe K, Tsunoda K, Itoh M et al (2013) Transglutaminase 2 and Factor XIII catalyze distinct substrates in differentiating osteoblastic cell line: utility of highly reactive substrate peptides. Amino Acids 44:209-214. doi:10.1007/s00726-011-1131-8

Yen J-H, Lin L-C, Chen M-C et al (2015) The metastatic tumor antigen 1-transglutaminase-2 pathway is involved in self-limitation of monosodium urate crystal-induced inflammation by upregulating TGF- $\beta 1$. Arthritis Res Ther. doi:10.1186/s13075-015-0592-7 